\title{
Ultrafast Electronic and Structural Phenomena in Graphite and Graphene
}

\author{
F. Carbone*, O.H. Kwon**, A. Cannizzo*, F. van Mourik*, M. Chergui*, and A.H. Zewail** \\ * Laboratory of Ultrafast Spectroscopy, ISIC, Ecole polytechnique fédérale de Lausanne, CH-1015 \\ Lausanne, Switzerland. \\ ** Physical Biology Center for Ultrafast Science and Technology, Arthur Amos Noyes Laboratory \\ of Chemical Physics, California Institute of Technology, Pasadena, CA 91125
}

Motion of ions and their interplay with bonded charge govern the functionality of materials. The scattering processes that undergo conduction carriers, such as electron-phonon or electron-electron interaction, influence their response to ultrafast stimuli such as time-varying electric fields or light pulses in terms of electrical mobility and bulk mechanics. Graphite and its derived materials, nanotubes, graphene, fullerenes, diamondoids, display a rich variety of electronic and mechanical properties which have found numerous exploitations in modern technology [1], and which are still stimulating intense research for future applications [2]. The basic structural and electronic dynamics of graphite have been widely investigated over 30 years, yet, by means of modern techniques, new phenomena have been observed in this simple but eclectic solid. When irradiated by femtosecond infrared pulses, coherent phonon generation has been observed in graphite [3]; also, diamond nanocrystals are formed on graphite surface [4], and at higher laser fluence, detachment of entire graphene flakes happens [5]. The dynamics of the electronic structure have been widely studied by means of ultrafast all-optical and photoelectron spectroscopy techniques [3-6], but a consensus on the characteristic timescales associated to carrier equilibration and electron-hole pair recombination processes hasn't been reached yet [7].

Here, we will present recent insights in the above-mentioned photoinduced structural and electronic phenomena in graphite. By means of a combination of ultrafast electron diffraction, ultrafast electron energy loss spectroscopy and transient optical absorption we deduced the structural motions precursor of the graphite to diamond transformation and graphene ablation, and their interplay with the conduction band carriers. We performed first-principle calculations on graphite electronic structure and simulate the effect of the observed structural dynamics on the ultrafast optical and plasmon response.

When illuminated by fs infrared pulses, the coherent motion of the carbon ions in the planar honeycombs is excited; as a result, a different stacking of graphene sheets is allowed and the crystal undergoes a compression. This behavior is pictorially displayed in Fig. 1, where the red arrows indicate the atomic motions induced by coherent phonon generation, and the yellow arrow indicates the resulting compression of the lattice. By means of ultrafast electron energy loss spectroscopy, the evolution of the charge density during such atomic motions was also observed, and revealed the tendency of the material to change the hybridization of orbitals from the typical graphite $s p^{2}$ to the three dimensional diamond $s p^{3}$. The charge distribution in compressed graphite and diamond are displayed in Fig. 2, where the distortion of the in-plane graphite hexagon during the formation of diamond is visible.

These atomic motions distort the material band structure, and as a result, the optical properties are modulated by structural effects. The separation of different contributions to the transient optical signal allowed us to determine the metallic nature of carrier relaxation in graphite, with an associated ultrafast time-scale of few hundreds fs, and deduce the pure response of charges in the isolated graphene layers. 


\section{References}

[1] M.S. Dresselhous et al., Graphite fibers and filaments, Springer-Verlag, New York, 1988.

[2] K.S. Novoselov et al., Science 306 (2004), 666.

[3] K. Ishioka et al., Phys. Rev. B 77, (2008) 121402(R).

[4] J. Kanasaki et al., Phys. Rev. Lett. 102, (2009) 087402.

[5] M. Lenner et al., Appl. Phys. Lett. 90 (2007) 153119.

[6] G. Moos et al., Phys. Rev. Lett 87, (2001) 267402.

[7] M. Breusing, et al., Phys. Rev. Lett. 102, (2009) 086809.

[5] This research was supported by the U.S.A. National Science Foundation and by the Gordon and Betty Moore center for physical biology at Caltech, and by the Suisse National Science foundation.

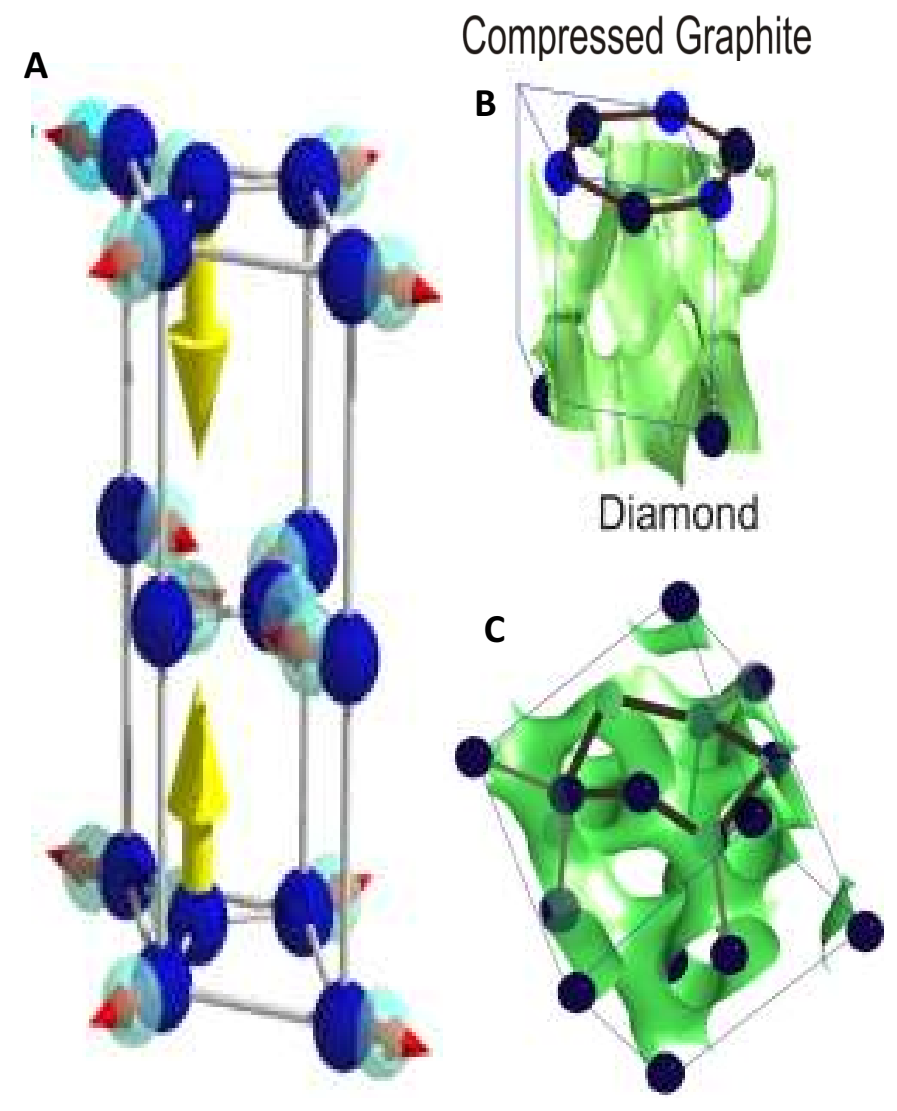

Fig. 1. A. Atomic motions and lattice compression in graphite. B. charge density distribution in compressed graphite. C. Charge density distribution in diamond. 